\title{
Tuberculose drogarresistente, orientações e acompanhamento pela atenção primária à saúde: revisão integrativa
}

\author{
Drug-resistant tuberculosis, guidance and monitoring by primary health care: an integrative \\ review
}

Tuberculosis farmacorresistente, orientación y seguimiento por parte de la atención primaria de salud: una revisión integradora

Sibele Naiara Ferreira Germano ${ }^{1 *}$, Arinete Véras Fontes Esteves ${ }^{1}$, Marlucia da Silva Garrido1.

\section{RESUMO}

Objetivo: Pesquisar as evidências na literatura científica, referentes às principais orientações e ao acompanhamento dos portadores de tuberculose drogarresistente na atenção primária à saúde. Métodos: Revisão integrativa da literatura com busca nas bases de dados MEDLINE/PubMed, CINAHL, LILACS e Scopus, de onde foram selecionados nove artigos científicos, incluídos nesta pesquisa. Resultados: Todos os artigos apresentaram orientações e acompanhamento do tratamento dos portadores de tuberculose drogarresistente. Destes, cinco destacaram a importância da adesão e do fortalecimento do regime terapêutico. Como acompanhamento, os artigos ressaltaram o tratamento diretamente observado, a participação de um conselheiro, o rastreamento ativo dos doentes e dos seus contatos domiciliares. Considerações finais: A pesquisa evidenciou orientações e acompanhamento efetivos para os casos de tuberculose drogarresistentena atenção primária à saúde, reforçando a importância do trabalho em equipe, incluindo o apoio psicológico, social e nutricional para a adesão ao tratamento e prevenção da transmissão de cepas resistentes na comunidade.

Palavras-chave: Aconselhamento, Atenção primária à saúde, Tuberculose resistente a múltiplos medicamentos, Continuidade da assistência ao paciente.

\begin{abstract}
Objective: To search for evidence in the scientific literature regarding the main guidelines and monitoring of drug-resistant tuberculosis patients in primary health care. Methods: Integrative literature review with search in the MEDLINE / PubMed, CINAHL, LILACS and Scopus databases, from which nine scientific articles were selected, included in this research. Results: All articles presented guidelines and monitoring of the treatment of drug-resistant tuberculosis patients. Of these, five highlighted the importance of adherence and strengthening the therapeutic regime. As a follow-up, the articles highlighted the treatment directly observed, the participation of a counselor, the active tracking of patients and their home contacts. Final considerations: The research showed effective guidance and monitoring for drug-resistant tuberculosis cases in primary health care, reinforcing the importance of teamwork, including psychological, social and nutritional support for treatment adherence and prevention of transmission of resistant strains in the community.
\end{abstract}

Keywords: Counseling, Primary health care, Tuberculosis, Multidrug-resistant, Continuity of patient care.

\section{RESUMEN}

Objetivos: Buscar evidencia en la literatura científica sobre las principales guías y seguimiento de los pacientes con tuberculosis farmacorresistente en la atención primaria de salud. Métodos: Revisión

1 Universidade Federal do Amazonas (UFAM), Manaus - AM. *E-mail: sibelenaiaraferreiragermano@gmail.com 
integradora de la literatura con búsqueda en las bases de datos MEDLINE / PubMed, CINAHL, LILACS y Scopus, de lãs cuales se seleccionaron nueve artículos científicos incluídos en esta investigación. Resultados: Todos los artículos presentaron pautas y seguimento del tratamiento de pacientes con tuberculosis farmacorresistente. De estos, cinco destacaron la importancia de la adherencia y el fortalecimiento del régimen terapéutico. Como seguimiento, los artículos destacaron el tratamiento directamente observado, la participación de un consejero, el seguimiento activo de los pacientes y sus contactos domiciliarios. Consideraciones finales: La investigación mostró una guía y un seguimiento eficaz de los casos de tuberculosis farmacorresistente en la atención primaria de salud, lo que refuerza la importância del trabajo en equipo, incluído el apoyo psicológico, social y nutricional para la adherencia al tratamiento y la prevención de la transmisión de cepas resistentes en la comunidad.

Palabras clave: Consejería, Primeros auxílios, Tuberculosis resistente a multiples medicamentos, Continuidad de la atención al paciente.

\section{INTRODUÇÃO}

Considerada uma enfermidade milenar, a tuberculose (TB) continua sendo uma das dez principais causas de morte por doença infecciosa no mundo, afetando cerca de 10 milhões de pessoas por ano. Entre os países com alta carga da doença, muitos estão longe do alcance da meta de eliminar este problema de saúde pública até 2035 (WHO, 2019).

A realização da busca ativa de sintomáticos respiratórios, diagnóstico e tratamento da tuberculose com o uso do esquema básico, é de competência da Atenção Primária à Saúde (APS). A cobertura deste nível de atenção, no Brasil, atingiu $70 \%$ da meta na detecção dos casos de tuberculose em 2015 , conforme proposto pela Organização Mundial da Saúde (OMS) (BERALDO AA, et al., 2017). Houve no ano de 2019 um incremento de $4 \%$ nos casos diagnosticados e acompanhados, mas não foram alcançadas as metas de cura de $85 \%$ e abandono menor que $5 \%$. Estes dados demonstram aumento da cobertura e do diagnóstico, mas evidenciam falha no acompanhamento do tratamento (BRASIL, 2020).

O abandono do tratamento da tuberculose no Brasil, em 2018, foi de $11,6 \%$ nos casos novos, proporção mais que duas vezes superior ao máximo de $5 \%$ recomendado pela Organização Mundial da Saúde (OMS). Ressalta-se que somente dois estados atingiram a meta estabelecida, com percentual inferior a $5 \%$ : Acre e Tocantins. Os maiores percentuais de abandono do tratamento foram registrados nas Unidades Federadas de Rondônia (20,1\%), Rio de Janeiro (15,5\%) e Amazonas (15,4\%) (BRASIL, 2020). Dentre as regiões brasileiras, Norte e Nordeste se destacaram com mais de 70\% de casos novos de tuberculose notificados e acompanhados pela APS no período de 2001 a 2019 (BRASIL, 2020).

Sabidamente, os casos de tuberculose têm cura quando diagnóstico e tratamento são realizados em tempo oportuno e corretamente, reduzindo a transmissão para outras pessoas. Esquemas de tratamentos inadequados, uso irregular da medicação e abandono do tratamento podem levar o doente a adquirir a tuberculose drogarresistente (RABAHI MF, et al., 2017).

O tratamento de tuberculose drogarresistente é mais longo e complexo, incluindo medicamentos de primeira e segunda linha, orais e injetáveis. Consequentemente, os portadores de tuberculose drogarresistente necessitam de atendimento em serviço especializado, ao mesmo tempo em que o acompanhamento diário deve ser realizado pela equipe da atenção primária à saúde, haja vista que este nível de atenção fica mais próximo, facilitando o Tratamento Diretamente Observado (TDO) para assegurar a adesão e prevenir a resistência aos antibióticos (WHO, 2019).

Devido ao fato de o tratamento da tuberculose drogarresistente ser de competência da atenção especializada, o foco dos profissionais da atenção primária voltou-se para o tratamento da tuberculose sensível, em detrimento do conhecimento sobre tuberculose drogarresistente, deixando uma falha no cuidado aos portadores desta doença neste nível de atenção, mostrando a necessidade da equipe multiprofissional estar sempre atualizada quanto ao tratamento e acompanhamento dos casos de tuberculose drogarresistente (RABAHI MF, et al., 2017). 
Um pilar essencial para o sucesso do Plano pelo fim da tuberculose no Brasil e no mundo é a orientação e acompanhamento adequado centrado no doente, com ênfase na adesão ao tratamento. $A$ adesão compreende não apenas a ingestão dos medicamentos, mas um processo dinâmico e multidimensional que envolve orientação e acompanhamento pela atenção primária à saúde, levando em consideração os aspectos comportamentais, psíquicos e sociais dos doentes, decisões e responsabilidades compartilhadas entre usuário, equipe de saúde e comunidade (BERALDO AA, et al., 2017).

As orientações efetivas trazem melhores resultados, por ajudar a dissipar dúvidas, crenças, mitos e promover a confiança e a empatia do doente, favorecendo o aumento da taxa de cura e prevenindo novos casos da doença. O conhecimento atualizado dos profissionais da atenção primária frente à tuberculose drogarresistente é essencial para realizar a orientação e o acompanhamento dos portadores desta doença, evitando desfechos desfavoráveis, por meio de estratégias assistenciais que favorecem o diagnóstico precoce, bem como o tratamento oportuno com vínculo e adesão. Neste momento destaca-se o importante papel dos profissionais de enfermagem no manejo dos casos de tuberculose e tuberculose drogarresistente, atuando nas ações de promoção da saúde, prevenção e tratamento da doença (BERALDO AA, et al., 2017). A maioria dos estudos disponíveis sobre tuberculose drogarresistente referem-se à epidemiologia, tratamento e mecanismos de mutação do Mycobacterium tuberculosis, com escassas publicações sobre orientações e acompanhamento dos portadores desta doença nos serviços de saúde, principalmente na atenção primária à saúde como coordenadora e ordenadora do cuidado.

Neste contexto justifica-se à necessidade de busca na literatura científica e publicação dos resultados obtidos para melhor orientação e acompanhamento dos portadores desta doença. Para suprir esta necessidade, a presente revisão teve como objetivo pesquisar as evidências, na literatura científica, referentes às principais orientações e ao acompanhamento dos portadores de tuberculose drogarresistente na atenção primária à saúde.

\section{MÉTODOS}

Trata-se de uma revisão integrativa da literatura que utiliza a síntese de múltiplos estudos científicos publicados para responder à questão norteadora da pesquisa a respeito da temática investigada. Para tanto, foram seguidas seis etapas bem definidas: $1^{\circ}$ ) definição da questão norteadora da pesquisa; $2^{\circ}$ ) busca na literatura atendendo os critérios de inclusão e exclusão definidos; $3^{\circ}$ ) categorização dos estudos; $4^{\circ}$ ) avaliação dos estudos incluídos na revisão; 5) interpretação dos resultados; 6ํ) apresentação da revisão integrativa (BOTELHO LLR, et al., 2011).

Para definição da questão norteadora desta pesquisa utilizou-se a estratégia PICo, que significa: PParticipantes, I- Fenômeno de Interesse e Co- Contexto (CARDOSO V, et al., 2019). Nesta pesquisa foi atribuído para P-portadores de tuberculose drogarresistente, I- orientações e acompanhamento, Co- atenção primária à saúde.

Essa atribuição resultou na seguinte questão de pesquisa: Quais as evidências, na literatura científica, referentes às orientações e ao acompanhamento dos portadores de tuberculose drogarresistente na atenção primária à saúde?

As buscas ocorreram em julho de 2020, nas bases de dados National Library of Medicine National Center of Biotechnology Information (MEDLINE/PubMed), Cumulative Index toNursingandAllied Health Literature(CINAHL), Literatura Latino-Americana e do Caribe em Ciências da Saúde (LILACS) e Scopus (Elsevier), acessadas pelo portal de periódicos da Coordenação de Aperfeiçoamento de Pessoal de Nível Superior (CAPES), via Comunidade Acadêmica Federada (CAFe).

Os artigos nas bases de dados foram acessados mediante a utilização dos seguintes Descritores em Ciências da Saúde (DeCS) e Medical Subject Headings (MeSH): Drug-Resistant Tuberculosis;Tuberculosis, Multidrug-Resistant; Counseling; Continuity of Patient Care; Primary Health Care.

Para estes descritores utilizaram-se os operadores booleanos "AND" e "OR" resultando nas seguintes estratégias de busca: Tuberculosis, Multidrug-Resistant" OR "Drug-Resistant Tuberculosis" AND 
Counseling OR "Continuity of Patient Care" AND "Primary Health Care"; "Tuberculosis, Multidrug-Resistant" OR "Drug-Resistant Tuberculosis" AND Counseling AND "Primary Health Care"; "Tuberculosis, MultidrugResistant" OR "Drug-Resistant Tuberculosis" AND "Continuity of Patient Care" AND "Primary Health Care".

Os critérios de inclusão foram: artigos disponíveis na integra (texto completo), online de acesso gratuito, disponíveis em português, inglês e/ou espanhol e publicados nos últimos seis anos (2014 a 2019). Os critérios de exclusão foram: estudos duplicados, estudos reflexivos, relatos de experiência, editorial e artigos que não responderam à questão norteadora de pesquisa.

Durante todo o processo de busca e seleção dos artigos, foi adotado o Preferred Reporting Items for Systematic reviews and Meta-Analyses (PRISMA), que ajudou no planejamento e organização do caminho percorrido para seleção criteriosa dos estudos e sua fidedignidade, assegurando o desenvolvimento de uma rigorosa revisão integrativa de literatura (GALVÃO TF, et al., 2015). Nas bases de dados foram encontrados 3.754 artigos utilizando-se a combinação dos descritores. Com a aplicação dos critérios de inclusão foram selecionados 1.380 artigos. Após nova triagem, em que foram aplicados os critérios de exclusão, selecionados os títulos e os resumos e eliminados os artigos em duplicidade, foram eleitos e lidos na íntegra 21 artigos.

Após leitura exaustiva, 12 desses artigos foram descartados, por não responderem à questão de pesquisa. Ao término da seleção, 9 artigos foram incluídos para análise e discussão desta revisão integrativa, conforme apresentado na Figura 1.

Estes artigos foram selecionados em três fases: na primeira, as buscas foram realizadas nas bases de dados mediante a utilização das estratégias selecionadas e aplicação dos critérios de inclusão; na segunda, em pares, os autores leram o título e o resumo de cada artigos identificado, aplicando os critérios de exclusão, a fim de separá-los para a fase seguinte; por fim, na terceira fase, foi feita a leitura na íntegra dos artigos, com o objetivo de selecionar para a análise aqueles que responderam à questão norteadora da pesquisa.

Figura 1 - Fluxograma de seleção dos artigos baseado no PRISMA.

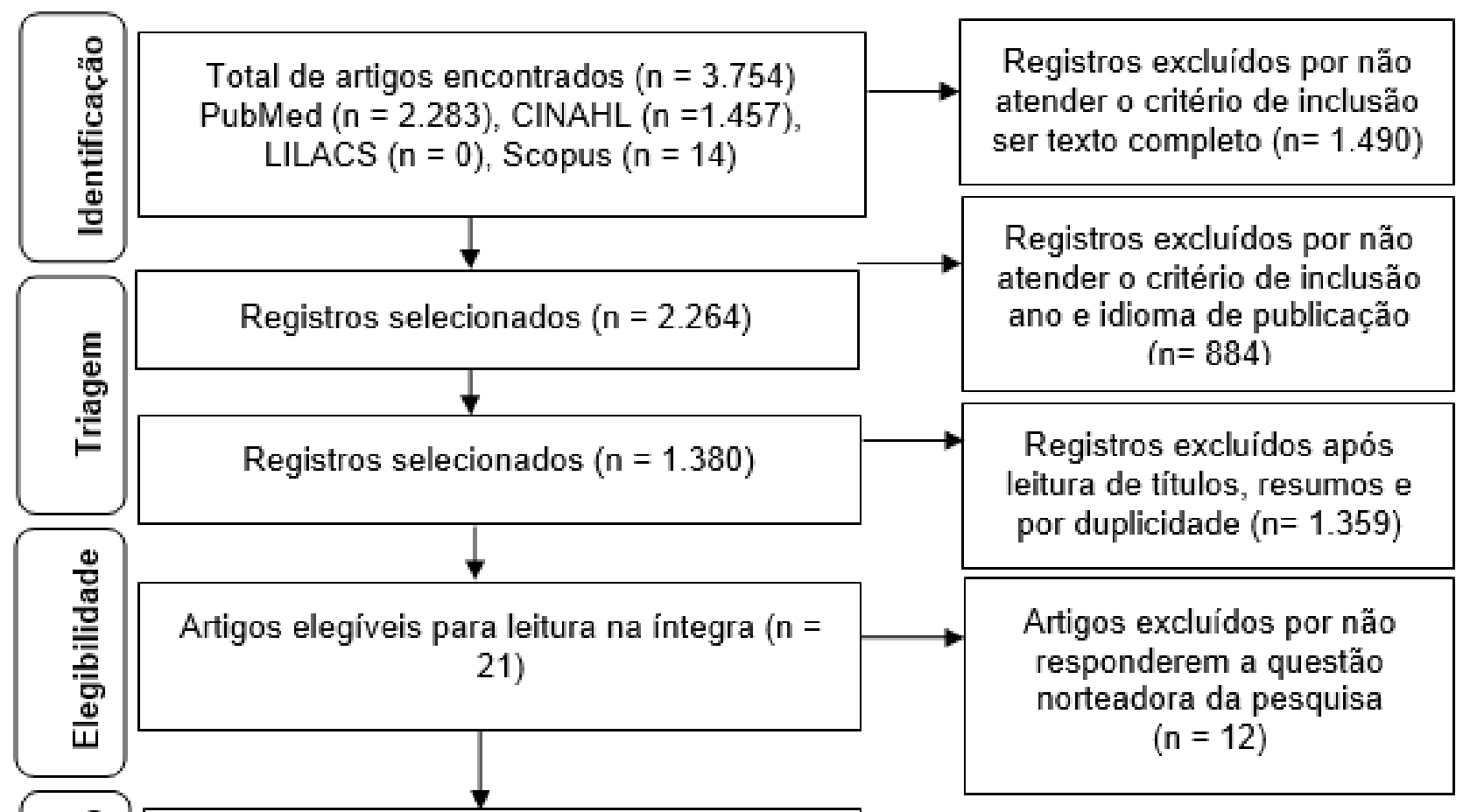

Estudos incluídos para síntese de revisão

$$
(n=9)
$$

Fonte: Germano SNF, et al., 2020. 


\section{RESULTADOS}

A amostra final, composta por nove artigos, traz orientações e informações sobre o acompanhamento dos portadores de tuberculose drogarresistente na atenção primária à saúde. Os artigos selecionados, escritos em diferentes países - um no Brasil (11,1\%), um em Myanmar (11,1\%), um no Peru (11,1\%), um no Sudão $(11,1 \%)$, dois no Nepal $(22,2 \%)$ e três na África do Sul $(33,3 \%)$, demonstram a distribuição das pesquisas publicados em diferentes países, os quais apresentam alta carga de tuberculose (Quadro 1).

No tocante à área de publicação dos artigos, destacou-se um na área da enfermagem $(11,1 \%)$, que corresponde ao cenário brasileiro, e os demais são internacionais (88,9\%), nas diversas áreas da saúde, incluindo a área de infectologia e de doenças pulmonares.

Quadro 1 - Caracterização dos artigos inclusos na revisão integrativa de literatura.

\begin{tabular}{|c|c|c|c|}
\hline $\begin{array}{l}\text { Autor/Ano/ } \\
\text { País }\end{array}$ & Periódico & Objetivo & $\begin{array}{l}\text { Método/Nível de } \\
\text { evidência }\end{array}$ \\
\hline $\begin{array}{l}\text { Ballestero } \\
\text { JGA, et al. } \\
(2014) \text { Brasil. }\end{array}$ & $\begin{array}{l}\text { Esc Anna } \\
\text { Nery. }\end{array}$ & $\begin{array}{c}\text { Analisar as vivências dos doentes de } \\
\text { tuberculose multirresistente sob a perspectiva } \\
\text { da integralidade da atenção. }\end{array}$ & $\begin{array}{l}\text { Qualitativo } \\
\text { Analítico } \\
\text { Transversal } \\
\text { Nível } 4 .\end{array}$ \\
\hline $\begin{array}{l}\text { Htun YM, et al. } \\
(2018) \\
\text { Myanmar. }\end{array}$ & $\begin{array}{l}\text { BMC Health } \\
\text { Serv Res. }\end{array}$ & $\begin{array}{l}\text { Explorar atrasos no diagnóstico e início do } \\
\text { tratamento e fatores associados entre os } \\
\text { portadores de tuberculose multirresistente. }\end{array}$ & $\begin{array}{l}\text { Descritivo Transversal } \\
\text { Nível } 4 .\end{array}$ \\
\hline $\begin{array}{l}\text { McNally TW, } \\
\text { et al. (2019) } \\
\text { Peru. }\end{array}$ & $\begin{array}{l}\text { BMC Health } \\
\text { Serv Res. }\end{array}$ & $\begin{array}{l}\text { Explorar as experiências e percepções dos } \\
\text { portadores de tuberculose multirresistente e os } \\
\text { profissionais de saúde envolvidos. }\end{array}$ & $\begin{array}{l}\text { Qualitativo } \\
\text { Nível } 4 .\end{array}$ \\
\hline $\begin{array}{l}\text { Ali MH, et al. } \\
\text { (2019) } \\
\text { Sudão. }\end{array}$ & $\begin{array}{l}\text { Expert Rev } \\
\text { Anti Infect } \\
\text { Ther. }\end{array}$ & $\begin{array}{l}\text { Avaliar os resultados do tratamento da } \\
\text { tuberculose multirresistentee preditores de } \\
\text { tratamentos desfavoráveis no Sudão. }\end{array}$ & $\begin{array}{l}\text { Estudo de Coorte } \\
\text { retrospectivo e } \\
\text { prospectivo } \\
\text { Nível } 4 . \\
\end{array}$ \\
\hline $\begin{array}{l}\text { Baral SC, et } \\
\text { al. (2014) } \\
\text { Nepal. }\end{array}$ & $\begin{array}{l}\text { BMC Public } \\
\text { Health. }\end{array}$ & $\begin{array}{l}\text { Identificar e documentar os problemas } \\
\text { enfrentados pelas pessoas que cuidam da } \\
\text { tuberculose multirresistente e informar o } \\
\text { desenvolvimento de estratégias. }\end{array}$ & $\begin{array}{l}\text { Qualitativo formativo e } \\
\text { explicativo } \\
\text { Nível } 4 .\end{array}$ \\
\hline $\begin{array}{l}\text { Walker IF, et } \\
\text { al. (2018) } \\
\text { Nepal. }\end{array}$ & PLOS ONE. & $\begin{array}{c}\text { Avaliar a viabilidade e aceitabilidade de um } \\
\text { pacote de apoio psicossocial para pessoas } \\
\text { recebendo tratamento para tuberculose } \\
\text { multirresistente. }\end{array}$ & $\begin{array}{l}\text { Quantitativo e } \\
\text { Qualitativo } \\
\text { Nível } 4 .\end{array}$ \\
\hline $\begin{array}{l}\text { Dudley L, et al. } \\
\qquad(2018) \\
\text { África do Sul. }\end{array}$ & PLOS ONE. & $\begin{array}{l}\text { Descrever a continuidade dos cuidados e } \\
\text { fatores de risco em portadores de tuberculose. }\end{array}$ & $\begin{array}{l}\text { Descritivo } \\
\text { observacional } \\
\text { retrospectivo } \\
\text { Nível } 4 . \\
\end{array}$ \\
\hline $\begin{array}{l}\text { Maraba N, et } \\
\text { al (2018) } \\
\text { África do Sul. }\end{array}$ & BMJ Open. & $\begin{array}{c}\text { Fazer uma intervenção piloto para determinar } \\
\text { se o apoio de um gerente de caso ajudaria os } \\
\text { adultos investigados para tuberculoes (TB) e } \\
\text { conectar os cuidados de TB e HIV. }\end{array}$ & $\begin{array}{l}\text { Estudo de Coorte } \\
\text { Nível } 4 .\end{array}$ \\
\hline $\begin{array}{l}\text { Cox H, et al. } \\
\quad(2014) \\
\text { África do Sul. }\end{array}$ & $\begin{array}{l}\text { Int } \mathrm{J} \text { Tuberc } \\
\text { Lung Dis. }\end{array}$ & $\begin{array}{c}\text { Descrever a detecção de casos e desfechos } \\
\text { do tratamento de TB-DR em um programa } \\
\text { baseado na comunidade. }\end{array}$ & $\begin{array}{l}\text { Retrospectivamente e } \\
\text { Prospectivamente } \\
\text { Nível } 5 .\end{array}$ \\
\hline
\end{tabular}

Fonte: Germano SNF, et al., 2020.

Com relação aos objetivos, as pesquisas analisados descrevem a continuidade dos cuidados pela atenção primária à saúde, trazendo vivências e percepções dos portadores de tuberculose drogarresistente em três estudos $(33,3 \%)$, problemas enfrentados pelos cuidadores desses doentes em um estudo $(11,1 \%)$, resultados do tratamento e preditores de tratamento desfavorável em $(22,2 \%)$, além de estratégias para orientação e acompanhamento dos que vivem com tuberculose drogarresistente $(33,3 \%)$. 
A maioria das pesquisas quarto utilizou a abordagem metodológica quantitativa $(44,4 \%)$, três $(33,3 \%)$ foram qualitativas, uma mista $(11,1 \%)$, e uma $(11,1 \%)$ não relatou a abordagem utilizada. Os tipos de estudos utilizados foram: dois estudos de coorte $(22,2 \%)$, um estudo analítico transversal $(11,1 \%)$, um descritivo transversal $(11,1 \%)$, um descritivo observacional retrospectivo $(11,1 \%)$, um formativo e explicativo $(11,1 \%)$, um retrospectivo e prospectivo $(11,1 \%)$ e outros dois $(22,2 \%)$ não descreveram o tipo de estudo.

Os níveis de evidência dos estudos inclusos nesta revisão foram analisados mediante a classificação da Agency for Healthcare Research and Quality (AHRQ) (GALVÃO CM, et al.,2003). Ao alcançarem níveis entre 4 e 5, devem produzir desenhos metodológicos com níveis de evidência mais fortes, para fundamentar a prática em saúde na temática.

Os nove artigos (100\%) trazem orientações necessárias aos portadores de tuberculose drogarresistente como: informações sobre a doença, diagnóstico, quadro clínico, efeitos colaterais, eficácia medicamentosa, coinfecção tuberculose (TB) e vírus da imunodeficiência humana (HIV), continuidade do acompanhamento, apoio emocional e cura. Destes, cinco $(55,5 \%)$ destacam a importância da adesão e do fortalecimento do regime terapêutico (Quadro 2).

Quadro 2 - Orientações e acompanhamento dos portadores de tuberculose drogarresistente pela atenção primária à saúde.

\begin{tabular}{|c|c|}
\hline Orientações & $\begin{array}{c}\text { Continuidade do atendimento ao portador da } \\
\text { doença/Acompanhamento }\end{array}$ \\
\hline Sobre o diagnóstico e adesão ao tratamento. & $\begin{array}{l}\text { Deve ser realizado pela Atenção Básica }(\mathrm{AB}) \text { próximo } \\
\text { ao doente com o acompanhamento do Tratamento } \\
\text { Diretamente Observado (TDO). }\end{array}$ \\
\hline $\begin{array}{l}\text { Mediante a educação em saúde abrangente, } \\
\text { sobre pré-teste, tratamento e provisão de } \\
\text { adesão com apoio emocional. }\end{array}$ & $\begin{array}{l}\text { Com rastreamento ativo da tuberculose e contatos } \\
\text { domiciliares. Realizando o exame Xpert na triagem, } \\
\text { fornecendo um pacote de apoio aos portadores desta } \\
\text { doença. }\end{array}$ \\
\hline $\begin{array}{l}\text { Educação em saúde com linguagem simples, } \\
\text { usando uma variedade de métodos e fontes de } \\
\text { informação, incluindo a Internet, sobre a } \\
\text { transmissão da tuberculose e da tuberculose } \\
\text { multirresistente, a adesão, efeitos colaterais e } \\
\text { eficácia dos medicamentos. }\end{array}$ & $\begin{array}{l}\text { Baseado na educação eficaz da população para } \\
\text { assumir a responsabilidade com sua própria saúde, } \\
\text { facilitando o envolvimento no tratamento e } \\
\text { incentivando a crença na medicina baseada em } \\
\text { evidências, além de fornecer uma boa nutrição com } \\
\text { apoio financeiro durante todo o tratamento. }\end{array}$ \\
\hline $\begin{array}{l}\text { Educação do doente sobre a adesão aos } \\
\text { diferentes regimes medicamentosos. }\end{array}$ & $\begin{array}{l}\text { Acompanhamento educacional, cobertura total do } \\
\text { fornecimento de medicamentos. }\end{array}$ \\
\hline $\begin{array}{l}\text { Aconselhamento individual do quadro de saúde } \\
\text { de cada doente, bem como combinado em } \\
\text { grupo para compartilhamento de vivências e } \\
\text { esclarecimento de dúvidas. }\end{array}$ & $\begin{array}{c}\text { Realizado com a participação de um conselheiro para } \\
\text { o aconselhamento individual e combinado dos } \\
\text { poratdores da doença, permitindo que expressassem } \\
\text { seus anseios e estigmas sofridos, além do apoio } \\
\text { financeiro fornecido. }\end{array}$ \\
\hline $\begin{array}{l}\text { Com materiais educativos sobre vários } \\
\text { aspectos da tuberculose multirresistente e seu } \\
\text { tratamento. }\end{array}$ & $\begin{array}{l}\text { Com triagem realizando aconselhamento individual } \\
\text { baseado na teoria da ativação comportamental. }\end{array}$ \\
\hline $\begin{array}{l}\text { Fornecer informações aos doentes e entre os } \\
\text { níveis de atenção sobre o quadro clínico e } \\
\text { tratamento da tuberculose. }\end{array}$ & $\begin{array}{c}\text { Acompanhamento com cuidado integrando aos } \\
\text { portadores de TB/HIV, realizando teste bacteriológico } \\
\text { e medicação. }\end{array}$ \\
\hline $\begin{array}{l}\text { Por contato ou por telefone/aplicativo, sobre o } \\
\text { exame de escarro, resultados do laboratório, } \\
\text { retorno à clínica para o tratamento e vínculo } \\
\text { tuberculose e HIV. }\end{array}$ & $\begin{array}{l}\text { Usando gerente de casos para acompanhar e apoiar } \\
\text { os doentes investigados para tuberculose e HIV. }\end{array}$ \\
\hline
\end{tabular}

Fonte: Germano SNF, et al., 2020.

Alguns desses artigos descrevem as etapas de desenvolvimento das orientações fornecidas, tendo três $(33,3 \%)$ utilizado a educação em saúde para orientação dos portadores de tuberculose drogarresistente, dois $(22,2 \%)$ recorreram ao método de aconselhamento individual e em grupo para compartilhamento de vivências e esclarecimento de dúvidas, um $(11,1 \%)$ utilizou materiais educativos, e o outro representando 
$(11,1 \%)$ empregou tecnologia móvel celular com aplicativo para entrar em contato com os doentes e prestar informações e orientações. Os demais estudos (22,2 \%) não informaram como foram realizadas as orientações.

Além das orientações, os artigos destacaram algumas formas de acompanhamento dos portadores da doença durante o tratamento na atenção primária à saúde. Dentre os artigos analisados, um $(11,1 \%)$ destacou o Tratamento Diretamente Observado (TDO), dois (22,2\%)trouxeram a participação de um conselheiro (gerente de caso), um $(11,1 \%)$ enfatizou o rastreamento ativo dos doentes e dos seus contatos domiciliares, realizando o exame Xpert, um $(11,1 \%)$ destacou a importânia da triagem com aconselhamento baseado na teoria da ativação comportamental, dois $(22,2 \%)$ destacaram o acompanhamento com cuidado integrado aos portadores de TB/HIV, fomentando as visitas domiciliares de rotina e apoio clínico e, por fim, a dois(22,2\%) referiram o acompanhamento pautado na educação eficaz da população, com cobertura total no fornecimento dos medicamentos e apoio financeiro.

\section{DISCUSSÃO}

A complexidade do tratamento dos portadores de tuberculose drogarresistente traduz-se na utilização de protocolos com associação de vários antibióticos por longo período, em média 18 meses. Ressalta-se que a medicação usada neste tratamento pode causar efeitos adversos, o que se constitui em um grande desafio à adesão destes doentes (WHO, 2019).

A interrupção do tratamento da tuberculose favorece o desenvolvimento da tuberculose drogarresistente, ocasionando falência do esquema de tratamento. Os níveis de resistência aos antibióticos usados no tratamento da doença podem variar de monorresistência a resistência extensiva. O tratamento da tuberculose resistente torna-se mais oneroso, além de registrar maior número de óbitos. Por isso, o crescente aumento do número de casos, apesar da luta contra a doença, tem preocupado os países globalmente (TSCHAMPL CA et al.,2016).

Neste cenário a atenção primária à saúde tem papel fundamental de promover a adesão ao tratamento, principalmente na realização do Tratamento Diretamente Observado (TDO) pelo profissional de saúde da atenção primária. Esta forma de tratamento vai além da observação da tomada diária dos medicamentos, indo ao encontro das necessidades dos doentes em seu contexto de vida, para esclarecer as dúvidas sobre diagnóstico e tratamento, possibilitando que a adesão se concretize como processo e não por imposição (BALLESTERO JGA et al., 2014).

Especificamente sobre o tratamento da tuberculose drogarresistente, o sucesso depende da colaboração da equipe de saúde mais próxima do doente, em parceria com o serviço de referência especializado. É necessário que o doente seja acolhido de forma humanizada pela rede de atenção à saúde, observando-se o seu contexto e singularidade, desde o diagnóstico até o final do tratamento. O estabelecimento de vínculo entre o profissional de saúde e o usuário requer a identificação de vulnerabilidades e necessidades, escuta e esclarecimentos de dúvidas que contribuirão para o sucesso do tratamento (BRASIL, 2018).

Cabe ainda à rede de atenção à saúde realizar o rastreamento sistematizado da tuberculose ativa, o monitoramento de todos os contatos domiciliares com teste rápido molecular para tuberculose e a investigação de resistência à rifampicina logo na triagemsão fatores importantes (HTUN YM, et al., 2018). Além disso, a ajuda financeira para o transporte e alimentação são essenciais para adesão ao tratamento (MCNALLY TW, et al., 2019). O diagnóstico precoce de resistência aos medicamentos é fundamental para a adesão ao tratamento eficaz, o que deve ser iniciado o mais cedo possível, evitando também os esquemas incorretos e sem qualquer benefício (ALENE KA, et al., 2017).

É também extremamente necessário fornecer aconselhamento psicológico para os doentes com diagnóstico de tuberculose drogarresistente, monitorando-os de perto quanto aos efeitos das drogas e das condições socioeconômicas, fornecendo suporte para uma alimentação saúdável durante todo o tratamento, especificamente nas fases iniciais (WALKER IF, et al., 2018). Orientações para a comunidade foram consideradas impactantes para minimizar os atrasos no diagnóstico e o tratamento dos usuários de baixa 
escolaridade, porque podem não valorizar a procura dos serviços de saúde, favorecendo a transmissão da doença na comunidade. Logo, os usuários bem orientados podem assumir a responsabilidade com sua própria saúde durante todo o período de tratamento até a cura (ALI MH, et al., 2019).

O Ministério da Saúde do Brasil recomenda que as equipes de saúde orientem o doente durante seu acompanhamento de forma clara, desde o primeiro contato, informando sobre a doença e o tratamento ao qual será submetido, as consequências do uso irregular da medicação, possíveis efeitos adversos e exame dos contatos (BRASIL, 2018).

Os resultados de dois estudos demonstraram a eficácia da utilização de conselheiros (gerentes de caso) para acompanhar e apoiar os portadores de tuberculose, fornecendo orientações sobre o exame de escarro, testagem para HIV, resultados de outros exames realizados e necessidade de retorno à clínica durante 0 tratamento de TB e TB/HIV (BARAL SC, et al., 2014; MARABA N, et al., 2018).

O acompanhamento dessa coinfecção também deve ser realizado de forma integrada com a atenção primária, mediante realização de testagem para HIV em todos os doentes de tuberculose, com orientação sobre a doença para os usuários e a comunidade, com vistas a reduzir o atraso no diagnóstico e assegurar tratamento adequado (DUDLEY L, et al., 108; TSCHAMPL CA, et al., 2016). Uma pesquisa recente realizado em uma unidade de referência no tratamento de TB DR na região nordestes do Brasil, traz o perfil epidemiológico dos casos de TB DR, demonstrando que a maioria dos indivíduos não realizam baciloscopia no final do tratamento, e dos que fizeram um quarto ainda deu positiva no final e acabaram abandonando a terapêutica.

Em relação ao teste de HIV, a maior parte obteve resultado negativo, contudo, mais de $30 \%$ da amostra não tinham realizado o exame. A pesquisa destaca a importância da adoção de estratégia para o aumento da adesão ao tratamento com acompanhamento correto do doente através da realização de exames com tomada da medicação realizando o TDO pela atenção primária mais próxima do doente, compartilhando assim o cuidado entre este nível de atenção e os demais níveis secundário e terciário (VALENÇA ÍMO, et al. 2020).

Por fim, todos os etudos trazemque a atenção primária à saúde deve ser corresponsável pelo acompanhamento dos casos de tuberculose drogarresistente, de forma compartilhada com as referências especializadas, fornecendo orientações efetivas ao doente e familiares, realizando o Tratamento Diretamente Observado, com visitas domiciliares de rotina, tratamento adequado e apoio clínico alinhados com as recomendações da Organização Mundial da Saúde, como estratégia essencial com o intuito de garantir a adesão cujo desfecho final almejado é a cura da doença (BALLESTERO JGA, et al., 2014).

A presente revisão integrativa teve limitação na obtenção dos resultados, devido às buscas nas bases de dados terem restringido os descritores tuberculose drogarresistente e atenção primária à saúde, e não ter incluído o descritor tuberculose, que é mais amplo e possui vários estudos sobre orientações e acompanhamento dos portadores desta doença neste nível de atenção. Isso reduziu o número de publicações disponíveis, demonstrando que o conhecimento científico com relação ao manejo de tuberculose drogarresistente na atenção primária ainda é incipiente, principalmente na área da enfermagem. Assim esta revisão traz importantes contribuições com evidências científicas da literatura, referente às principais orientações e acompanhamento dos portadores desta doença na atenção primária à saúde.

\section{CONSIDERAÇÕES FINAIS}

A pesquisa evidenciou orientações essenciais que devem ser prestadas aos portadores de tuberculose drogarresistente, bem como a importância do acompanhamento efetivo dos mesmos na atenção primária à saúde, onde deverão ser realizadas outras ações complementares para o controle da doença, tais como, o tratamento diretamente observado, exame de contatos e baciloscopias de controle mensal até o final do tratamento. Os artigos também reforçaram a importância do trabalho em equipe, incluindo o apoio psicológico, social e nutricional para a adesão ao tratamento e prevenção da transmissão de cepas resistentes para a comunidade. 


\section{REFERÊNCIAS}

1. ALENE KA, et al. Treatment outcomes in patients with multidrug-resistant tuberculosis in north-west Ethiopia. Trop Med Inter Health. 2017; 22 (3): 351-62.

2. ALI MH, et al. Assessment of multidrug-resistant tuberculosis (MDR-TB) treatment outcomes in Sudan; findings and implications. Expert Rev Anti Infect Ther. 2019; 17 (11): 927-37.

3. BALLESTERO JGA, et al. Multidrug-resistant tuberculosis: integral healthcare from the discourse analysis perspective. Esc Anna Nery. 2014; 18 (3): 515-21.

4. BARAL SC, et al. The importance of providing counselling and financial support to patients receiving treatment for multi-drug resistant TB: mixed method qualitative and pilot intervention studies. BMC Public Health. 2014; $14: 46$.

5. BERALDO AA, et al. Adherence to tuberculosis treatment in Primary Health Care: perception of patients and professionals in a large municipality. Esc Anna Nery. 2017; 21 (4): e20170075.

6. BOTELHO LLR, et al. O método da revisão integrativa nos estudos organizacionais. Gest Socied. $2011 ; 5$ (11): 121 36.

7. BRASIL. Ministério da Saúde (BR). Manual de Recomendações para o Controle da Tuberculose no Brasil [Internet]. Brasília: Ministério da Saúde; 2018.

8. BRASIL. Ministério da Saúde (BR). Secretaria de Vigilância em Saúde. Boletim Epidemiológico de Tuberculose [Internet]. Brasília: Ministério da Saúde; 2020.

9. CARDOSO V, et al. Systematic review of mixed methods: method of research for the incorporation of evidence in Nursing. Texto Contexto Enferm. 2019; 28: e20170279.

10. CERVANTES J. Tuberculosis. Digging deep in the soul of humanity. Respir Med. 2016; 119: $20-2$.

11. $\mathrm{COX} \mathrm{H}$, et al. Community-based treatment of drug-resistant tuberculosis in Khayelitsha, South Africa. Int $\mathrm{J}$ Tuberc Lung Dis. 2014; 18 (4): 441-8.

12. DUDLEY L, et al. Mind the gap! Risk factors for poor continuity of care of TB patients discharged from a hospital in the Western Cape, South Africa. PLoS One. 2018; 13 (1): e0190258.

13. GALVÃO CM, et al. A busca das melhores evidências. Rev Esc Enferm USP. 2003; 37 (4): 43-50.

14. GALVÃO TF, et al. Principais itens para relatar revisões sistemáticas e metanálises: a recomendação PRISMA. EpidemiolServ Saúde. 2015; 24 (2): 335-42.

15. HTUN YM, et al. Delay in diagnosis and treatment among adult multidrug resistant tuberculosis patients in Yangon Regional Tuberculosis Center, Myanmar: a cross-sectional study. BMC Health Serv Res. 2018; $18: 87$.

16. MARABA N, et al. Linkage to care among adults being investigated for tuberculosis in South Africa: pilot study of a case manager intervention. BMJ Open.2018; 8: e021111.

17. MCNALLY TW et al. Improving outcomes for multi-drugresistant tuberculosis in the Peru vi an Amazon - a qualitative study exploring the experiences and perceptions of patient sand health care professionals. BMC Health Serv Res.2019; 19: 594.

18. RABAHI MF, et al. Tuberculosis treatment. J Bras Pneumol. 2017; 43 (5): 472-86.

19. TSCHAMPL CA, et al. Use of transnational services to prevent treatment interruption in tuberculosis-infected persons who leave the United States. Emerg Infect Dis. 2016; 22 (3): 417-25.

20. VALENÇA ÍMO, et al. Perfil epidemiológico dos casos de tuberculose drogarresistente. Revista Eletrônica Acervo Saúde REAS/EJCH. 2020; 56: e4334.

21. WALKER IF, et al. Implementation of a psychosocial support package for people receiving treatment for multidrugresistant tuberculosis in Nepal: A feasibility and acceptability study. PLoS One. 2018;13 (7): e0201163.

22. WHO. WORLD HEALTH ORGANIZATION. Global tuberculosis report 2019 [Internet]. Geneva: França: WHO/CDS/TB; 2019. 